\title{
OPTIMAL CONTROL COMPUTATION TO ACCOUNT FOR ECCENTRIC MOVEMENT
}

\author{
L. S. JENNINGS ${ }^{1}$, K. H. WONG ${ }^{2}$ and K. L. TEO ${ }^{1}$
}

(Received 25 August 1994; revised 8 March 1995)

\begin{abstract}
A class of optimal control models which involve different weightings in the integrand of the objective function is considered. The motivation for considering this class of problems is that this type of objective function is used to account for eccentric movement in biomechanical models. The computation of these optimal control problems using control parametrization directly is difficult, firstly because of ill-conditioning, and secondly because the objective function is not differentiable. A method for smoothing the integrand is presented with convergence results. An example is computed which shows favourable computational improvements.
\end{abstract}

\section{Introduction}

Optimal control models of animal movement involve an objective function usually expressed as an integral over a time period of a function (the integrand) which represents some physical quantity such as power, work, torque, etc. References [5] [7] contain examples of such objective functions. Many of these objective function integrands are not differentiable (at some values of time) with respect to the control (torque) and state (angular position and velocity) variables, either because of an absolute value being taken or because the absolute value function is being weighted with one if the argument is positive and one third if the argument is negative. This latter operation represents a 'lopsided' absolute value function, and is used to account for eccentric movement, that is, to account for the difference between the cases of velocity in the same direction as the torque and velocity in the opposite direction to the torque.

The computation of these optimization problems using the control parametrization technique proves difficult, firstly because they are ill conditioned, and secondly

\footnotetext{
${ }^{1}$ Department of Mathematics, University of Western Australia, Nedlands 6009, Australia

${ }^{2}$ Department of Applied Mathematics,, University of Witwatersrand, South Africa

(C) Australian Mathematical Sóciety, 1996, Serial-fee code 0334-2700/96 
because of these points in time of non-differentiability of the integrands. We address the latter problem in this paper. The numerical evaluation of the integrals is prone to almost random errors of sizeable magnitude, as most quadrature methods require a high degree of smoothness of the integrand to attain their theoretical accuracy. This in turn affects the optimization algorithm in terms of convergence as these codes assume function values are accurate to a greater accuracy than the quadratures can produce for a non-smooth integrand. The integration of the costate dynamics is also affected by the non-differentiability but to a lesser extent. These points of non-differentiability are in general unknown, so it is very difficult to account for them in any automatic quadrature or numerical integration method. Hence we employ smoothing techniques as a general purpose technique to overcome the computational problems and to enable the production of convergence results for the method as part of the control parametrization technique. The smoothing of the absolute value function is reported in [8]. Another smoothing technique may be found in [1]. This current paper covers the case of a 'lopsided' absolute value function.

This paper proceeds directly to the smoothing technique employed and then produces a sequence of convergence results which relate the new results of this paper to previous results for state constraints and the control parametrization method. Finally a realistic worked example is presented.

\section{The problem statement}

Consider a process described by the initial value dynamics on $[0, T]$,

$$
\dot{x}(t)=f(t, x(t), u(t)), \quad x(0)=x^{0},
$$

where the state variables $x \in \mathbb{R}^{n}$ and the control variables $\boldsymbol{u} \in \mathbb{R}^{r}$. Assume there are upper and lower bounds on the controls for all $t \in[0, T]$, so that $u(t):[0, T] \rightarrow U$, where $U$ is a compact and convex set defined by

$$
U=\left\{v \in \mathbb{R}^{r}: \alpha_{i} \leq v_{i} \leq \beta_{i}, \quad i=1, \ldots, r\right\} .
$$

A bounded measurable function $\boldsymbol{u}$ from $[0, T]$ into $\mathbb{R}^{r}$ is said to be an admissible control if $u(t) \in U$ for almost all $t \in[0, T]$. Let $\mathscr{U}$ be the class of all such admissible controls.

For each $\boldsymbol{u} \in \mathscr{U}$, let $\boldsymbol{x}(\cdot \mid \boldsymbol{u})$ be the corresponding solution of the system (2.1). The inequality terminal state constraints and the inequality continuous state constraints are defined, respectively, as follows:

$$
\phi_{i}(x(T \mid u)) \geq 0, \quad i=1, \ldots, N_{T},
$$


where $\phi_{i}, i=1, \ldots, N_{T}$, are real valued functions defined on $\mathbb{R}^{n}$, and,

$$
g_{i}(t, x(t \mid u)) \geq 0, \quad \forall t \in[0, T], \quad i=1, \ldots, N,
$$

where $g_{i}, i=1, \ldots, N$, are real valued functions defined on $[0, T] \times \mathbb{R}^{n}$. Let $\mathscr{F}$ be the set that consists of all those elements from $\mathscr{U}$ such that the constraints (2.2) and (2.3) are satisfied. Elements from $\mathscr{F}$ are called the feasible controls and $\mathscr{F}$ is called the class of feasible controls.

PROBLEM P. Given the system (2.1), find a control $u \in \mathscr{F}$ such that the cost functional

$$
J(u)=\int_{0}^{T} a\left(g_{0}(t, x(t \mid u), u(t))\right) d t,
$$

is minimized over $\mathscr{F}$, where

$$
a(g)= \begin{cases}c_{1} g, & \text { if } g \geq 0, \\ -c_{2} g, & \text { otherwise, }\end{cases}
$$

is similar to the absolute value function but with different slope magnitudes either side of the origin. The function $g_{0}$ is real valued and $c_{1}$ and $c_{2}$ are positive constants.

We assume that the following conditions are satisfied:

(A1) $f:[0, T] \times \mathbb{R}^{n} \times \mathbb{R}^{r} \rightarrow \mathbb{R}^{n}$ is piecewise continuous on $[0, T]$ for each $(\boldsymbol{x}, \boldsymbol{u}) \in$ $\mathbb{R}^{n} \times \mathbb{R}^{r}$ and is continuously differentiable with respect to each of the components of $\boldsymbol{x}$ and $\boldsymbol{u}$ for each $t \in[0, T]$; furthermore, there exists a constant $K>0$ such that

$$
|f(t, x, u)| \leq K(1+|x|),
$$

where $|\cdot|$ denotes the Euclidean norm;

(A2) for each $i=1, \ldots, N_{T}, \phi_{i}: \mathbb{R}^{n} \rightarrow \mathbb{R}$ is continuously differentiable;

(A3) for each $i=1, \ldots, N, g_{i}:[0, T] \times \mathbb{R}^{n} \rightarrow \mathbb{R}$ is continuously differentiable;

(A4) $g_{0}:[0, T] \times \mathbb{R}^{n} \times \mathbb{R}^{r} \rightarrow \mathbb{R}$ is piecewise continuous on $[0, T]$ for each $(\boldsymbol{x}, \boldsymbol{u}) \in$ $\mathbb{R}^{n} \times \mathbb{R}^{r}$ and is continuously differentiable with respect to each of the components of $\boldsymbol{x}$ and $\boldsymbol{u}$ at each $t \in[0, T]$.

These assumptions are standard for the solution of optimal control problems using the control parametrization method (see [9]). They guarantee the existence of solutions to (2.1), and the existence of co-state variables.

REMARK 2.1. From the theory of differential equations, we recall that (2.1) admits a unique solution $\boldsymbol{x}(\cdot \mid \boldsymbol{u})$, corresponding to each $u \in L_{\infty}^{r}$ and hence for each $\boldsymbol{u} \in \mathscr{U}$. There exists a bounded subset $\mathscr{X}$ of $\mathbb{R}^{n}$ such that $\boldsymbol{x}(t \mid \boldsymbol{u}) \in \mathscr{X}$ for all $t \in[0, T]$ and for all $u \in \mathscr{U}$. If $\left\{\boldsymbol{u}^{p}\right\}_{p=1}^{\infty}$ is any sequence of functions in $\mathscr{U}$ that converges to a function $u$ a.e. on $[0, T]$, then $\lim _{p \rightarrow \infty}\left\|x\left(\cdot \mid u^{p}\right)-x(\cdot \mid u)\right\|_{\infty}=0$, and $\lim _{p \rightarrow \infty} J\left(u^{p}\right)=J(u)$. 


\section{Control parametrization}

The control parametrization method summarized in [9] is used to solve problem $P$. Each control is approximated by a piecewise constant control based on a partition of the interval $[0, T]$. Let $\left\{I_{p}\right\}_{p=1}^{\infty}$ be a sequence of partitions of the interval $[0, T]$ such that $I_{p}$ has $n_{p}+1$ points, $I_{p+1}$ is a refinement of $I_{p}$ and that the maximum length of a subinterval tends to zero as $p \rightarrow \infty$. Let $\mathscr{U}^{p}$ be the subset of admissible controls which are piecewise constant and consistent with partition $I_{p}$. Hence every control $\boldsymbol{u}^{p} \in \mathscr{U}^{p}$ can be uniquely identified with a set of $r n_{p}$ parameters $\sigma^{p}$ and vice versa. Let $\mathscr{F}^{p}$ be the subset of $\mathscr{U}^{p}$ which are feasible, that is, satisfy (2.2) and (2.3). Let $\Theta^{p}$ be the set of parameters $\sigma^{p}$ which correspond to those piecewise constant controls from $\mathscr{U}^{p}$. Similarly, let $\Xi^{p}$ be the set of parameters $\sigma^{p}$ corresponding to those controls from $\mathscr{F}^{p}$. Restricting the control parameters to $\Xi^{p}$, the system (2.1) becomes

$$
\dot{x}(t)=\hat{f}\left(t, x(t), \sigma^{p}\right), \quad x(0)=x^{0},
$$

where $\hat{f}$ is obtained from $\boldsymbol{f}$ in the obvious way. For each $\sigma^{p} \in \Xi^{p}$, let $x\left(\cdot \mid \sigma^{p}\right)$ be the corresponding solution of the system (3.1). Similarly the constraints (2.2) and (2.3) become

$$
\phi_{i}\left(\boldsymbol{x}\left(T \mid \boldsymbol{\sigma}^{p}\right)\right) \geq 0, \quad i=1, \ldots, N_{T},
$$

and

$$
g_{i}\left(t, x\left(t \mid \sigma^{p}\right)\right) \geq 0, \quad \forall t \in[0, T], \quad i=1, \ldots, N
$$

respectively.

We define an approximate problem $\mathrm{P}(p)$ as:

Problem $\mathrm{P}(p)$. Find a control $\sigma^{p} \in \Xi^{p}$ such that the cost functional

$$
\hat{J}\left(\sigma^{p}\right)=\int_{0}^{T} a\left(\hat{g}_{0}\left(t, x\left(t \mid \sigma^{p}\right), \sigma^{p}\right)\right) d t
$$

is minimized over $\Xi^{p}$, where $\hat{g}_{0}$ is obtained from $g_{0}$ in the obvious way. This is an optimization problem over $\mathbb{R}^{r n_{p}}$.

Note that convergence of general discrete approximations for constrained minimization is the subject matter of [2]. 


\section{Smoothing the cost functional}

Because the integrand in (3.4) is not differentiable we need to smooth the corner of the 'lopsided absolute value' function $a(g)$. Of the many ways of doing this we choose the following because it has a minimum at the same place as the original function. The smooth approximation is

$$
a^{\delta}(g)= \begin{cases}c_{1} g, & \text { if } c_{1} g>\delta, \\ \left(\delta^{2}+\left(c_{1} g\right)^{2}\right) / 2 \delta, & \text { if } 0 \leq c_{1} g \leq \delta, \\ \left(\delta^{2}+\left(c_{2} g\right)^{2}\right) / 2 \delta, & \text { if }-\delta \leq c_{2} g<0, \\ -c_{2} g, & \text { if } c_{2} g<-\delta .\end{cases}
$$

In view of (A4) and Remark 2.1, it is clear that the function $a^{\delta}\left(\hat{g}_{0}(t, x, \sigma)\right)$ possesses the following properties:

(i) it is piecewise continuous on $[0, T]$ for each $(\boldsymbol{x}, \boldsymbol{\sigma}) \in \mathbb{R}^{n} \times \mathbb{R}^{r n_{p}}$ and is continuously differentiable with respect to each of the components of $x$ and $\sigma$ for each $t \in[0, T]$,

(ii) for each $(t, \boldsymbol{x}, \boldsymbol{\sigma}) \in[0, T] \times \mathbb{R}^{n} \times \mathbb{R}^{r n_{p}}$

$$
0 \leq a^{\delta}\left(\hat{g}_{0}(t, \boldsymbol{x}, \boldsymbol{\sigma})\right)-a\left(\hat{g}_{0}(t, \boldsymbol{x}, \boldsymbol{\sigma})\right)<\delta,
$$

(iii) for each $t \in[0, T], \sigma^{*}$ minimizes $a\left(\hat{g}_{0}(t, \boldsymbol{x}(t \mid \boldsymbol{\sigma}), \boldsymbol{\sigma})\right)$ if and only if it minimizes $a^{\delta}\left(\hat{g}_{0}(t, x(t \mid \sigma), \sigma)\right)$.

By virtue of these properties, $\left.a^{\delta}\left(\hat{g}_{0}\right)\right)$ is an ideal approximation of the nonsmooth function $a\left(\hat{g}_{0}\right)$. We define an approximate problem to $\mathrm{P}(p)$.

PRoBlem $\mathrm{P}^{\delta}(p)$. Find a control $\sigma^{p} \in \Xi^{p}$ such that the cost functional

$$
\hat{J}^{\delta}\left(\boldsymbol{\sigma}^{p}\right)=\int_{0}^{T} \boldsymbol{a}^{\delta}\left(\hat{g}_{0}\left(t, \boldsymbol{x}\left(t \mid \boldsymbol{\sigma}^{p}\right), \boldsymbol{\sigma}^{p}\right)\right) d t,
$$

is minimized over $\Xi^{p}$.

THEOREM 4.1. Let $\sigma^{p, \delta}$ and $\sigma^{p}$ be optimal controls to problems $P^{\delta}(p)$ and $P(p)$ respectively. Then

$$
0 \leq \hat{J}\left(\sigma^{p, \delta}\right)-\hat{J}\left(\sigma^{p}\right)<\delta T
$$

Proof. By virtue of property (ii) of the function $a^{\delta}\left(g_{0}(t, \boldsymbol{x}, \boldsymbol{\sigma})\right)$ we have

$$
\hat{J}^{\delta}\left(\sigma^{p}\right)<\hat{J}\left(\sigma^{p}\right)+\delta T .
$$


Since $\sigma^{p, \delta}$ is an optimal control of the problem $\mathrm{P}^{\delta}(p)$ and $\sigma^{p}$ is a feasible control of the problem $\mathrm{P}^{\delta}(p)$, we have

$$
\hat{J}^{\delta}\left(\sigma^{p, \delta}\right) \leq \hat{J}^{\delta}\left(\sigma^{p}\right)<\hat{J}\left(\sigma^{p}\right)+\delta T .
$$

Using property (ii) again and (4.3) we have

$$
\hat{J}\left(\sigma^{p, \delta}\right) \leq \hat{J}^{\delta}\left(\sigma^{p, \delta}\right)<\hat{J}\left(\sigma^{p}\right)+\delta T .
$$

The fact that $\sigma^{p}$ is an optimal control of problem $\mathrm{P}(p)$ and $\sigma^{p, \delta}$ is a feasible control of problem $\mathrm{P}(p)$ implies that

$$
0 \leq \hat{J}\left(\boldsymbol{\sigma}^{p, \delta}\right)-\hat{J}\left(\boldsymbol{\sigma}^{p}\right)<\delta T .
$$

THEOREM 4.2. Let $\left\{\sigma^{p, \delta}\right\}$ be a sequence in $\delta$ of optimal controls of approximate problems $P^{\delta}(p)$. Then there exists an accumulation point of the sequence $\left\{\sigma^{p, \delta}\right\}$ for $\delta \rightarrow 0$. Furthermore any accumulation point is an optimal control to the problem $P(p)$.

PROOF. From the definition of $\Theta^{p}$, it is clear that the sequence $\left\{\sigma^{p, \delta}\right\}$ is bounded in the Euclidean norm of $\mathbb{R}^{r n_{p}}$. Thus there exists an accumulation point $\overline{\boldsymbol{\sigma}}^{p}$, and a subsequence which is again denoted by the original sequence, such that

$$
\lim _{\delta \rightarrow 0}\left|\sigma^{p, \delta}-\tilde{\boldsymbol{\sigma}}^{p}\right|=0 .
$$

Using Remark 2.1 and (4.4), we have

$$
\lim _{\delta \rightarrow 0} \hat{J}\left(\sigma^{p, \delta}\right)=\hat{J}\left(\overline{\boldsymbol{\sigma}}^{p}\right) .
$$

Using Remark 2.1, (A2) and (A3), it is clear that $\overline{\boldsymbol{\sigma}}^{p}$ is a feasible control of the problem $\mathrm{P}(p)$. Thus if $\sigma^{p}$ is an optimal control of the problem $\mathrm{P}(p)$ we have

$$
\hat{J}\left(\overline{\boldsymbol{\sigma}}^{p}\right) \geq \hat{J}\left(\sigma^{p}\right) .
$$

Now in view of (4.2), we have

$$
\lim _{\delta \rightarrow 0}\left[\hat{J}\left(\sigma^{p, \delta}\right)-\hat{J}\left(\sigma^{p}\right)-\delta T\right] \leq 0 .
$$

From (4.5) and (4.7) we get

$$
\hat{J}\left(\overline{\boldsymbol{\sigma}}^{p}\right) \leq \hat{J}\left(\sigma^{p}\right) .
$$

We conclude from (4.7) and (4.8) that $\bar{\sigma}^{p}$ is also an optimal control of the problem $\mathrm{P}(p)$. 


\section{Continuous state constraint approximation}

For completeness of this paper we outline the results of [10] on the method for solving problems with continuous state constraints, as it affects our convergence results. There are some further assumptions about the constraints which are given in [10]. The method is to replace constraints (2.3) with the integral constraints

$$
G_{i}\left(\boldsymbol{\sigma}^{p}\right)=\int_{0}^{T} \min \left(0, g_{i}\left(t, \boldsymbol{x}\left(t \mid \boldsymbol{\sigma}^{p}\right)\right)\right) d t=0, i=1, \ldots, N,
$$

which in turn are replaced by

$$
G_{i}^{\epsilon}\left(\sigma^{p}\right)=\int_{0}^{T} s^{\epsilon}\left(g_{i}\left(t, x\left(t \mid \sigma^{p}\right)\right)\right) d t+\tau(\epsilon) \geq 0, \quad, i=1, \ldots, N .
$$

The function $s^{\epsilon}(g)$ smooths the function $\min (0, g)$ as follows,

$$
s^{\epsilon}(g)= \begin{cases}g, & \text { if } g \leq-\epsilon, \\ -(g-\epsilon)^{2} / 4 \epsilon, & \text { if }-\epsilon<g<\epsilon, \\ 0, & \text { if } g \geq \epsilon .\end{cases}
$$

The parameter $\tau$, dependent on $\epsilon$, is used to keep the control parameter vector within $\Xi^{p}$. To this end, we introduce another approximate problem, dependent on $\epsilon$ with the view to letting $\epsilon \rightarrow 0$. Let $\mathscr{G}^{p}$ be the subset of $\Theta^{p}$ which satisfies (3.2) and (5.1).

Problem $\mathrm{P}^{\delta, \epsilon}(p)$. Find a control $\sigma^{p} \in \mathscr{G}^{p}$ such that the cost functional (4.1) is minimized over $\mathscr{G}^{P}$.

Lemma 3.3 of [10] shows that by choosing $\tau$ small enough $\mathscr{G}^{p} \subset \Xi^{p}$, and we restate this result.

LEMMA 5.1. There exists $\tau(\epsilon)>0$ such that for all $\tau, 0<\tau<\tau(\epsilon)$, any feasible control $\sigma^{p, \delta, \epsilon}$ of the problem $P^{\delta, \epsilon}(p)$ is also a feasible control of the problem $P^{\delta}(p)$. That is, for all positive $\tau<\tau(\epsilon)$ if

$$
G_{i}^{\epsilon}\left(\sigma^{p, \delta, \epsilon}\right) \geq-\tau, \quad i=1, \ldots, N,
$$

then

$$
G_{i}\left(\sigma^{p, \delta, \epsilon}\right)=0, \quad i=1, \ldots, N
$$

REMARK 5.1. Algorithm A2 of [10] can be modified to generate a sequence $\left\{\sigma^{p, \delta, \epsilon}\right\}$ of control parameters in $\epsilon$ so that each element is in the feasible region of $\mathrm{P}^{\delta}(p)$. The next theorem is a direct consequence of Theorem 4.1 of [10]. 
THEOREM 5.1. Let $\left\{\sigma^{p, \delta, \epsilon}\right\}$ be a sequence in $\epsilon$ of the suboptimal control vectors produced by Algorithm A2 of [10]. Then

$$
\lim _{\epsilon \rightarrow 0} \hat{J}^{\delta}\left(\boldsymbol{\sigma}^{p, \delta, \epsilon}\right)=\hat{J}^{\delta}\left(\boldsymbol{\sigma}^{p, \delta}\right),
$$

where $\sigma^{p, \delta}$ is an optimal control vector of the problem $P^{\delta}(p)$. Furthermore, any accumulation point of $\left\{\sigma^{p, \delta, \epsilon}\right\}$ is a solution of the problem $P^{\delta}(p)$.

\section{Convergence results as $p \rightarrow \infty$}

We consider the convergence properties of the sequence in $p$ of approximate optimal controls of the problem $\mathrm{P}(p)$ to the true optimal control of the problem $\mathrm{P}$. The following theorems are direct consequences of Theorem 4.2 and 4.3 of [10].

THEOREM 6.1. Let $\sigma^{p, *}$ be the optimal parameter of the approximate problem $P(p)$, and let $\boldsymbol{u}^{p, *}$ be the corresponding piecewise constant control. Suppose that $\boldsymbol{u}^{*}$ is an optimal control of the problem $P$. Then

$$
\lim _{p \rightarrow \infty} J\left(\boldsymbol{u}^{p, *}\right)=J\left(\boldsymbol{u}^{*}\right)
$$

THEOREM 6.2. Let $u^{p, *}$ be as defined in Theorem 6.1. Suppose that

$$
\lim _{p \rightarrow \infty} \boldsymbol{u}^{p, *}=\overline{\boldsymbol{u}}, \quad \text { a.e. on }[0, T] .
$$

Then $\overline{\boldsymbol{u}}$ is an optimal control of the problem $P$.

This completes the convergence results, covering the cases $\epsilon \rightarrow 0, \delta \rightarrow 0$ and $p \rightarrow \infty$, and shows that in these limits the control function converges to an optimal control of the original problem. In practice, computational limitations mean that the smallest values of $\delta$ and $\epsilon$ are about $10^{-4}$ and that $n_{p}$, the number of control parameters representing a single control, is large at 40 . Most optimal control problems become more ill-conditioned if $n_{p}$ is allowed to become larger.

\section{Eccentric movement}

We chose an example of a four-segment leg (as of the extinct moa of New Zealand) executing a swing through movement. The data is supplied by Dr R.N. Marshall and represents a guess of the bio-data. The state equations of motion are as in [6], relating the angular acceleration of segments to the torque generated between 
segments by muscle, and the states are the angular positions $\left(\theta_{i}\right)$ and velocities $\left(\omega_{i}\right)$ of the segments. Here, we consider the case involving 4 planar segments, leading to a system of 8 ordinary differential equations. These differential equations are similar to those describing the dynamics of a planar 4-link robotic system. They are highly nonlinear. The relevant data of segment length, centre of mass, mass and moment of inertia are given in Table 1, as are the initial values of the states at time $t=0,\left(\theta^{0}, \omega^{0}\right)$, and the final values of the states at $t=T=0.36$ seconds, $\left(\theta^{T}, \omega^{T}\right)$.

TABLE 1. Bio-data.

\begin{tabular}{|c|cccccccc|}
\hline segment & length & c.of m. & mass & m.of I. & $\theta_{i}^{0}$ & $\omega_{i}^{0}$ & $\theta_{i}^{T}$ & $\omega_{i}^{T}$ \\
\hline 1 & 0.100 & 0.050 & 0.70 & 0.069 & 5.24 & -0.3 & 5.74 & -0.5 \\
2 & 0.215 & 0.108 & 1.40 & 0.148 & 4.71 & -0.5 & 5.50 & -0.3 \\
3 & 0.470 & 0.204 & 4.25 & 0.258 & 4.19 & -0.3 & 5.24 & -0.1 \\
4 & 0.290 & 0.126 & 8.40 & 0.157 & 5.32 & 0.4 & 5.67 & 0.0 \\
\hline
\end{tabular}

We are not in this paper defending the data in Table 1, or the differential equation model in [6], but rather wish to concentrate on algorithm performance. The objective function chosen for this study is the integral over time of muscle power transferred into segments taking into account eccentric work:

$$
J=\int_{0}^{0.36} \sum_{i=1}^{4}\left\{\begin{array}{cc}
\tau_{i} \omega_{i}, & \text { if } \tau_{i} \omega_{i} \geq 0 \\
-\frac{1}{3} \tau_{i} \omega_{i}, & \text { otherwise }
\end{array}\right\} d t
$$

where $\tau_{i}$ is the torque generated by segment $i$ and is a control variable. The constraints of reaching the final state values are treated using a terminal time equality constraint, namely,

$$
\phi_{1}(\theta(T), \omega(T))=\sum_{i=1}^{4}\left(\theta_{i}(T)-\theta_{i}^{T}\right)^{2}+\sum_{i=1}^{4}\left(\omega_{i}(T)-\omega_{i}^{T}\right)^{2}=0 .
$$

Because this is a sum of squares of quantities required to be zero, the optimization software must be able to reduce $\phi_{1}$ to a very small value. No constraints of the type (2.3) are required in this computation, but sometimes they are necessary to stop the first segment rotating through more than $180^{\circ}$. The initial guess of the torque values over time is zero for all time, and the minimum and maximum allowable values for the torques are given by $\alpha=(-9,-20,-60,-60)$ and $\beta=(9,25,60,90)$.

We use the MISER3 optimal control software ([3],[4]) on three cases, two without smoothing and one with smoothing of the objective integrand. MISER3 has the option of stopping the optimization algorithm after a preset number of iterations so that the user can decide whether to continue as if we did not stop (warm start), or to cold 
start, that is, to allow the optimization routine to reset itself and begin again from the current point. If the optimization software runs into trouble we can cold start the computation. One run without smoothing is allowed to do only warm starts every 50 iterations, which means that it executes as if no limit is placed on the number of iterations. The other run without smoothing is cold started about every 200 iterations in an attempt to improve the optimization performance. This technique is helpful in ill-conditioned optimization problems. (The large number of iterations required is due to ill-conditioning.) We report the progress of the three cases at every 50 iterations (or fewer if the optimization failed) giving the objective value and the constraint value and $\delta$ in the third case (smoothing).

TABLE 2. Case 1. No smoothing, no operator intervention.

\begin{tabular}{|c|c|c|c|}
\hline cold/warm & Iteration no. & Objective & Constraint \\
\hline c & 50 & 33.07 & $2.3 \times 10^{-1}$ \\
w & 100 & 37.56 & $1.1 \times 10^{-5}$ \\
w & 150 & 32.72 & $4.6 \times 10^{-7}$ \\
w & 200 & 31.11 & $3.8 \times 10^{-8}$ \\
w & 250 & 29.91 & $2.8 \times 10^{-7}$ \\
w & 300 & 28.73 & $2.2 \times 10^{-8}$ \\
w & 350 & 28.10 & $4.1 \times 10^{-9}$ \\
w & 400 & 27.72 & $1.8 \times 10^{-9}$ \\
w & 450 & 27.59 & $3.8 \times 10^{-10}$ \\
w & 500 & 27.51 & $1.6 \times 10^{-10}$ \\
\hline
\end{tabular}

It is clear that Case 3 gives the better objective value even though the optimization failed twice. The cold starts marked with an asterix indicate a cold start forced on the iteration by the failure of the optimization routine. Such failure is usually because of too many function values computed on a line search or because an uphill search direction has been used. Without operator intervention the non-smoothed case stops well short of the value achieved by the smooth case, and even much coaxing by the operator still does not give as good a result as the smoothed case. In the non smoothed case, even though the function value changes little after 450 iterations, the 'solution' is but a set of values giving a smaller objective function value than the starting values. This can of course be said of all three solutions, in that a numerical procedure will only attempt to find a local minimum. In ill-conditioned optimization small changes in objective function values are not a measure of being close to the point giving the minimum. Another feature of an ill-conditioned optimization problem is that large 
TABLE 3. Case 2. No smoothing, operator intervention.

\begin{tabular}{|c|c|c|c|}
\hline cold/warm & Iteration no. & Objective & Constraint \\
\hline c & 50 & 33.07 & $2.3 \times 10^{-1}$ \\
w & 100 & 37.56 & $1.1 \times 10^{-5}$ \\
w & 150 & 32.72 & $4.6 \times 10^{-7}$ \\
w & 200 & 31.11 & $3.8 \times 10^{-8}$ \\
c & 205 & 30.84 & $7.7 \times 10^{-5}$ \\
c $^{*}$ & 255 & 28.38 & $3.6 \times 10^{-5}$ \\
w & 305 & 27.22 & $7.6 \times 10^{-7}$ \\
w & 355 & 26.41 & $8.3 \times 10^{-8}$ \\
w & 405 & 25.98 & $1.7 \times 10^{-8}$ \\
c & 409 & 25.98 & $9.0 \times 10^{-7}$ \\
c $^{*}$ & 421 & 24.87 & $3.5 \times 10^{-3}$ \\
c $^{*}$ & 471 & 23.13 & $7.5 \times 10^{-3}$ \\
w & 521 & 26.48 & $1.7 \times 10^{-7}$ \\
w & 571 & 26.19 & $1.5 \times 10^{-8}$ \\
w & 621 & 25.97 & $4.1 \times 10^{-9}$ \\
w & 671 & 25.91 & $5.7 \times 10^{-10}$ \\
w & 699 & 25.89 & $1.4 \times 10^{-10}$ \\
\hline
\end{tabular}

changes in parameters with small changes in objective values is common, even within the tangent plane of the constraints.

\section{Conclusions}

This paper reports on a smoothing technique for a non-differentiable objective function. An algorithm has been developed for the successful computation of an optimal control problem. The method has been used to solve an optimal control problem arising from a consideration of eccentric movement in biomechanics. Convergence properties of the algorithm are given. The underlying problem of ill conditioning which occurs in many optimal control problems of biomechanics has not been tackled in this paper but will be the subject of further work. There are so-called regularization techniques which can be used to tackle this problem.

\section{Acknowledgement}

This work was supported by the Australian Research Council. 
TABLE 4. Case 3. Smoothing.

\begin{tabular}{|c|c|c|c|c|}
\hline cold/warm & Iteration no. & $\delta$ & Objective & Constraint \\
\hline c & 50 & $10^{-2}$ & 40.64 & $2.2 \times 10^{-2}$ \\
w & 100 & $10^{-2}$ & 34.18 & $2.0 \times 10^{-5}$ \\
w & 126 & $10^{-3}$ & 31.59 & $8.3 \times 10^{-6}$ \\
c $^{*}$ & 176 & $10^{-3}$ & 29.72 & $3.1 \times 10^{-5}$ \\
w & 195 & $10^{-3}$ & 28.36 & $1.0 \times 10^{-5}$ \\
c $^{*}$ & 245 & $10^{-3}$ & 27.71 & $6.3 \times 10^{-6}$ \\
w & 295 & $10^{-4}$ & 26.36 & $3.8 \times 10^{-7}$ \\
w & 345 & $10^{-4}$ & 25.93 & $1.6 \times 10^{-7}$ \\
w & 395 & $10^{-4}$ & 25.60 & $8.8 \times 10^{-9}$ \\
w & 445 & $10^{-4}$ & 25.51 & $7.1 \times 10^{-10}$ \\
w & 495 & $10^{-4}$ & 25.46 & $4.7 \times 10^{-10}$ \\
w & 545 & $10^{-4}$ & 25.44 & $6.8 \times 10^{-11}$ \\
\hline
\end{tabular}

\section{References}

[1] B. D. Craven, "Nondifferentiable optimization by smooth approximation", Optimization 17 (1986) 3-17.

[2] B. D. Craven, "Convergence of discrete approximations for constrained minimization", J. Austral. Math. Soc. Ser. B 36 (1994) 50-59.

[3] L. S. Jennings, M. E. Fisher, K. L. Teo and C. J. Goh, "MISER3, optimal control software, theory and user manual", EMCOSS, Perth WA, 1990.

[4] L. S. Jennings, M. E. Fisher, K. L. Teo and C. J. Goh, "MISER3: Solving optimal control problems — an update", Adv. Eng. Software and Workstations 13 (1991) 190-196.

[5] R. N. Marshall and L. S. Jennings, "Performance objectives in the stance phase of human pathological walking", Human Movement Sci. 9 (1990) 599-611.

[6] R. N. Marshall, R. K. Jensen and G. A. Wood, "A general Newtonion simulation of an $n$-segment open chain model", J. of Biomech. 18 (1985) 359-367.

[7] R. N. Marshall, G, A. Wood and L. S. Jennings, "Performance objectives in human movement: A review and application to the stance phase of normal walking", Human Movement Sci. 8 (1989) 571-594.

[8] K. L. Teo and C. J. Goh, “On constrained optimization problems with non-smooth cost functionals”, Appl. Math. Opt. 17 (1988) 181-190.

[9] K. L. Teo, C. J. Goh and K. H. Wong, A Unified Computational Approach to Optimal Control Problems (Longman Scientific and Technical, UK, 1991).

[10] K. L. Teo and L. S. Jennings, "Nonlinear optimal control problems with continuous state inequality constraints", J. Opt. Theory and Appl. 63 (1989) 1-22. 